\title{
Revealing the multidimensional mental representations of natural objects underlying human similarity judgments
}

\author{
Martin N. Hebart ${ }^{1,2^{*}}$, Charles Y. Zheng ${ }^{3}$, Francisco Pereira ${ }^{3}$, \& Chris I. Baker ${ }^{1}$ \\ ${ }^{1}$ Laboratory of Brain and Cognition, National Institute of Mental Health, National Institutes of \\ Health, Bethesda, MD, USA \\ ${ }^{2}$ Vision and Computational Cognition Group, Max Planck Institute for Human Cognitive and \\ Brain Sciences, Leipzig, Germany \\ ${ }^{3}$ Machine Learning Core, National Institute of Mental Health, National Institutes of Health, \\ Bethesda, MD, USA
}

*Correspondence should be addressed to Martin Hebart: hebart@cbs.mpg.de

\begin{abstract}
Objects can be characterized according to a vast number of possible criteria (e.g. animacy, shape, color, function), but some dimensions are more useful than others for making sense of the objects around us. To identify these "core dimensions" of object representations, we developed a data-driven computational model of similarity judgments for real-world images of 1,854 objects. The model captured most explainable variance in similarity judgments and produced 49 highly reproducible and meaningful object dimensions that reflect various conceptual and perceptual properties of those objects. These dimensions predicted external categorization behavior and reflected typicality judgments of those categories. Further, humans can accurately rate objects along these dimensions, highlighting their interpretability and opening up a way to generate similarity estimates from object dimensions alone. Collectively, these results demonstrate that human similarity judgments can be captured by a fairly lowdimensional, interpretable embedding that generalizes to external behavior.
\end{abstract}




\section{Introduction}

We live in a world full of objects that we can identify, place into different categories, communicate and reason about, and act on in a meaningful manner. These abilities are remarkable, given that our ever-changing environment requires us to constantly map unique sensory information from the things around us to our internal representations of objects, categories, and concepts. In order to carry out this mapping and make sense of our world, we therefore need to determine the similarity between the sensory information emanating from the environment and our internal mental representations. Not surprisingly, similarity has been suggested to play an important role in elucidating the structure of our mental representations and can help explain how we recognize objects ${ }^{1,2}$, form categories ${ }^{3-5}$, structure our conceptual knowledge $^{6-8}$, and predict the behavior of our visual world based on our experience ${ }^{9}$. Moreover, representational similarities offer a useful tool to relate behavior, computational models, and brain activity patterns ${ }^{10}$.

Despite the success of similarity and the wide use of similarity judgments for studying mental representations of objects, similarities alone offer only an indirect and mostly descriptive view of the format of our mental representations. They can inform us about the degree with which two or more representations are similar, but are agnostic as to what properties - or dimensions each representation is made up of and what dimensions are shared between those representations. For example, most people would agree that a dog and a cow are more similar than a dog and a car, probably because dogs and cows share more relevant dimensions, such as being animate or natural, or soft. To understand the structure of our mental representations of objects, we need to identify those core dimensions that form the basis of our similarity judgments. These dimensions need to fulfill two criteria. First, they should be predictive of behavior and thus able to characterize the mental representational space. Second, to move beyond description and provide understanding, we need to identify a set of dimensions from the infinite number possible, that can be interpreted meaningfully.

Here, we present a computational model of mental representations of objects based on a largescale assessment of human similarity judgments for natural object images. Prior experimental, neuropsychological, and neuroimaging evidence have led to the proposal of object dimensions such as animacy, manipulability, or real-world size ${ }^{11-13}$, but they only describe a selective and largely incomplete portion of our mental representational space. In contrast to traditional smallscale experimental approaches that often use artificial stimuli or words ${ }^{14}$, we collected a large number of similarity judgments for images of 1,854 different objects, capturing both visual and conceptual mental representations for a wide, representative range of natural objects. Rather than relying on explicit verbal reports of what object features are perceived as being relevant ${ }^{15,16}$, the model learns those dimensions directly from these similarity judgments.

Using this data-driven approach, we identify 49 dimensions underlying similarity judgments that lead to excellent prediction of both single trial behavior and similarity scores between pairs of objects. We demonstrate that the dimensions are meaningful and characterize the large-scale structure of our mental representations of objects. The model allows for the accurate prediction 
of categorization behavior, while within categories individual dimensions reflect object typicality. Finally, we demonstrate that human participants can use these dimensions directly to provide good predictions of similarity judgments, underscoring the interpretability of dimensions and offering a first step towards a generative model of perceived similarity of natural objects.

\section{Results}

To characterize the representational space of natural objects, we had to overcome several obstacles. First, we needed to identify a set of objects that is representative of the objects encountered in the real world. For that purpose, we chose the 1,854 objects in the THINGS database ${ }^{17}$, which we developed to provide a comprehensive list of living and non-living things according to their everyday use in the American English language. For each object, we chose a representative image that had been shown to be named consistently during the creation of this database. The advantage of using images rather than words is that they may provide additional purely perceptual information that is relevant for judging the similarity of objects and that might not come to mind immediately when using words.

Second, we needed to identify a task that would allow us to best quantify the similarity between pairs of objects. Ideally, this task would highlight all relevant dimensions contributing to the similarity of pairs of objects and would be independent of the context in which these objects appear ${ }^{3,18,19}$. While pairwise similarity ratings on a Likert scale are one of the most popular approaches, this task implicitly assumes that all dimensions relevant to judging the similarity of pairs of objects are always and immediately available to the observer, even when the objects are very dissimilar and may seem to have nothing in common. Here we chose a different approach, in which we concurrently presented three object images $i, j$ and $k$ in a triplet odd-oneout task (Fig. 1a). By choosing the odd-one-out object, participants indicate which pair of objects $(i, j),(i, k)$, or $(j, k)$ is the most similar among this set. The key benefit of this task is that the third object always serves as a context for the other two objects, thus highlighting the relevant dimensions that make two objects most similar. By repeatedly varying the third object for a given pair of objects, we are thereby implicitly sampling across a wide range of contexts in which the objects might be encountered. We can then express similarity as an approximation of the probability $\mathrm{p}(i, j)$ of participants choosing objects $i$ and $j$ together, irrespective of context. In addition, since the similarity of objects is determined with respect to all other objects, this naturally constrains the number of possible dimensions to those relevant for discriminating among objects ${ }^{20}$.

Third, we needed to collect sufficient data with these objects and this task. While the odd-oneout task provides a principled approach for investigating similarity across contexts, for 1,854 objects it would require $\sim 1.06$ billion combinations of triplet judgments for a single estimate of the full similarity matrix. This would make conducting the odd-one-out task at this scale not feasible. However, if similarity depends only on a small number of independent dimensions, it should be possible to approximate the entire similarity matrix with only a fraction of those judgments. In this study, we sampled 1.46 million unique responses from 5,301 workers using 
the online platform Amazon Mechanical Turk (Fig. 1a), pooling all responses across workers (median number of responses per worker: 60 ). This corresponded to $0.14 \%$ of possible unique trials.

a
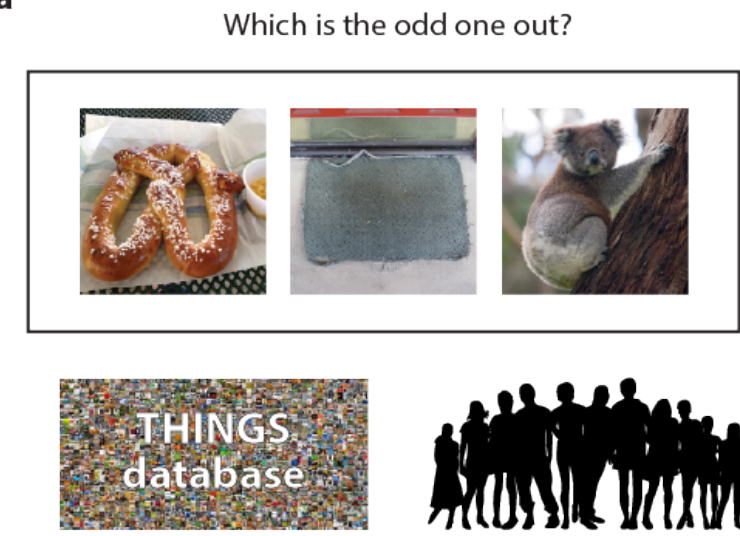

broad sampling of images

(1,854 objects)

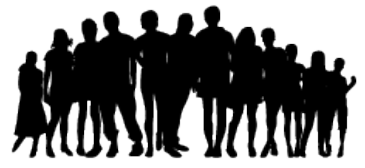

large-scale online crowdsourcing $(\mathrm{n}=1.46$ million trials)

b

\author{
Representational \\ embedding
}

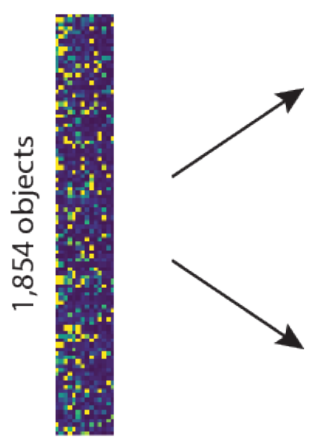

object dimensions
Predicted choice behavior

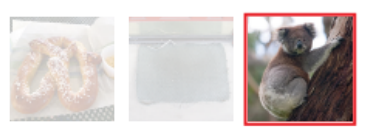

Predicted object similarity

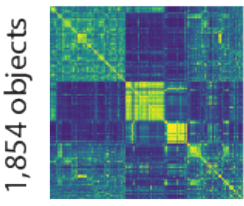

1,854 objects

C

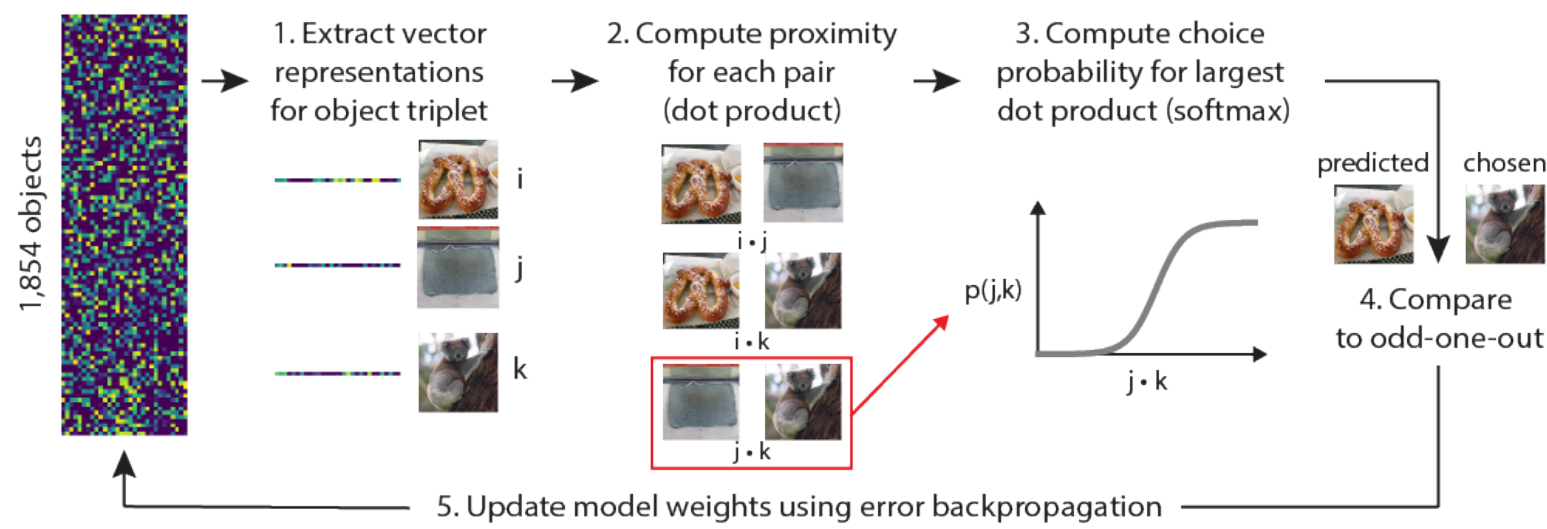

Fig. 1 | Task and modeling procedure for large-scale identification of mental object representations. a We applied a triplet odd-one-out similarity task to images of the 1,854 objects in the THINGS database ${ }^{17}$ and collected a large number of ratings (1.46 million) using online crowdsourcing. The triplet odd-one-out task measures object similarity as the probability of choosing two objects together. This task evokes different minimal contexts as a basis for grouping objects together, which in turn emphasizes the relevant dimensions. $\mathbf{b}$ The goal of the modeling procedure was to learn an interpretable representational embedding that captures choice behavior in the odd-one-out task and predicts object similarity across all pairs of objects. Since only a subset of all possible triplets had been sampled $(0.14 \%$ of 1.06 billion possible combinations), this model additionally served to complete the sparsely sampled similarity matrix. c The model reflects the assumed cognitive process underlying the odd-one-out task. The embedding was initialized with random weights and would carry out predictions for which object pair was the most similar, based on the dot product. The prediction of the most similar pair is equivalent to predicting the remaining object as the odd-one-out. Model predictions were initially at chance (see example for a prediction that deviates from the choice) but learned gradually to predict behavioral choices. To allow for error backpropagation to the weights, the model was implemented as a shallow neural network. 
Our goal was to build a computational model that is capable of predicting behavior in the oddone-out task, that captures the similarity between all pairs of objects, and that provides interpretable object dimensions. At the center of this model is a representational embedding, which is a quantitative characterization of objects as vectors in a multidimensional representational space. This embedding can be described as a matrix $X$, in which each column corresponds to a dimension and each row to an object vector across all dimensions (Fig. 1b). In the context of our model, this embedding should allow us to (1) predict behavior for individual trials not included in the training data and (2) generate the entire similarity matrix between all pairs of objects.

To create this embedding, we made two key assumptions. First, we assumed that dimensions are sparse, which is a reasonable assumption, given that not all dimensions are expressed in all objects. For example, for a putative dimension of animacy, a cardboard box would likely have a value of 0 . Second, we assumed that dimensions are continuous and positive. Accordingly, the numeric value of an object for a given dimension could then be interpreted as the degree to which the dimension is expressed in the object, which should support interpretability ${ }^{21,22}$.

The modeling procedure was as follows (Fig. 1c). We initialized the model with 90 random dimensions, assuming that after model fitting, sparsity would reduce the dimensionality of the embedding to a smaller number. For a given triplet $(i, j, k)$ for which we had collected a behavioral judgment, we then calculated the dot product between the embedding vectors of all three pairs of objects $(i, j),(i, k)$, and $(j, k)$. Accordingly, when two objects express high values for many dimensions, this measure yields a large number, while when one object expresses high values in dimensions for which the other object expresses low values, this measure yields a small number. Next, based on those three dot products, we estimated the probability of choosing one of the three pairs of objects in this context, which is equivalent to the third object being the odd-one-out. To this end, we used the softmax function which has been demonstrated to be suitable for relating representational proximity to similarity in the context of choice models and for estimating generalization behavior ${ }^{20,23}$. Finally, the difference between the predicted choice probability and the actual choice served as a model prediction error, which allowed us to adapt the model dimensions in proportion to this error (see Methods for details on this optimization procedure). The model was trained on $90 \%$ of the available trials, and the remaining $10 \%$ were later used for an independent assessment of model performance (see below).

\section{A stable and predictive model of behaviorally measured similarity}

As expected, due to the sparsity constraint, many of the 90 initial dimensions revealed values close to 0 and were discarded, leaving us with 49 dimensions. We then sorted the dimensions based on the sum of all dimension values across all objects, in descending order. Due to the stochastic nature of the modeling procedure, fitting the model repeatedly may lead to a different embedding and a slightly different number of dimensions. To estimate the stability of the model, we re-ran it 20 times with different random initializations (see Methods). Across those models, most dimensions exhibited high reproducibility (Pearson $r>0.9$ in 34/49 dimensions, Pearson $r$ 
$>0.6$ in 46/49 dimensions), demonstrating that the procedure generated a highly stable and reproducible embedding (see Extended Data Figure 1 for a plot of the reproducibility of all dimensions). There was a strong correlation between the ranks of the dimensions and the dimension reproducibility (Spearman's $\rho: 0.75, p<0.001$, randomization test, $95 \% \mathrm{Cl}: 0.61$ 0.85 ), indicating that reproducibility of individual dimensions was driven mostly by their overall importance in the model.

Having demonstrated the reproducibility of the model dimensions, we next tested the predictive performance of the model. First, we estimated how well we could predict individual choices in the odd-one-out task using trials from the independent test set. To gain an understanding of the best possible prediction any model could achieve for these 1,854 objects given the variation present in the data ("noise ceiling"), we additionally sampled 1,000 randomly-chosen triplets 25 times and estimated the consistency of choices for each triplet across participants. Averaged across those triplets, the upper limit in fitting individual trial behavior from the data was $67.22 \%$ $( \pm 1.04 \%)$. Overall, the model correctly predicted $64.60 \%( \pm 0.23 \%)$ of individual trials in the independent test data (Fig. 2a). This means that the model achieved $92.25 \%( \pm 1.50 \%)$ of the best possible accuracy at predicting behavior, demonstrating excellent predictive performance at the individual trial level given the noise in the data.

a Behavioral prediction

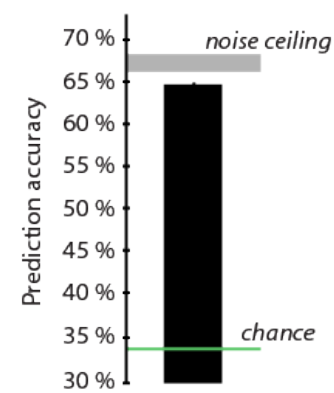

b

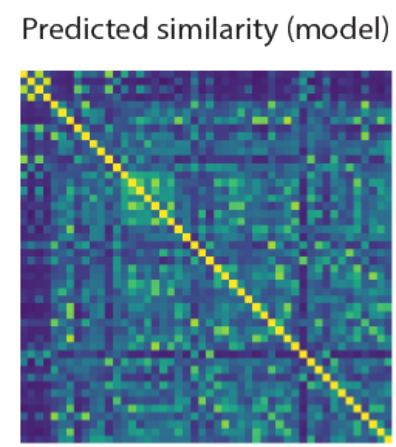

48 diverse objects

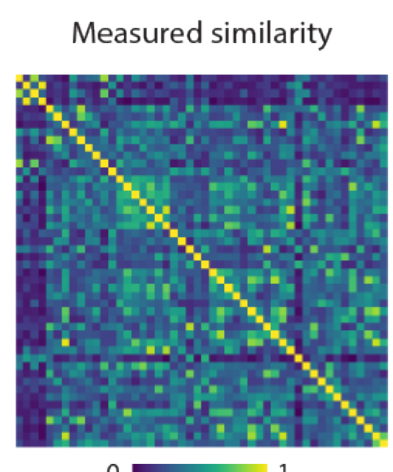

0

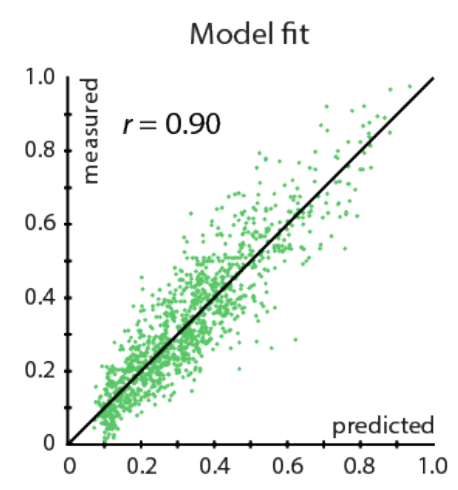

Fig. 2 | Predictiveness of the computational model for single trial behavioral judgments and similarity. a Model performance was evaluated by predicting choice behavior at the individual trial level. The noise ceiling denotes the maximal performance any model could achieve given the noise in the data and is determined by the consistency in participants' responses to the same triplet. The performance of the model in predicting independent test data approached noise ceiling, demonstrating excellent predictive performance. Error bars and shaded areas denote $95 \%$ confidence intervals. b To estimate how well the model predicted behavioral similarity, a model-generated similarity matrix was compared to a fullysampled behavioral similarity matrix for 48 diverse objects. Results reveal a close fit (Pearson $r=0.90, p$ $<0.001$, randomization test, $95 \% \mathrm{Cl}: 0.88-0.91$ ), demonstrating that most explainable variance was captured by the model.

To evaluate how well the model could predict behaviorally measured similarity, we next generated a fully-sampled similarity matrix of 48 diverse objects and compared it to the similarity matrix predicted by our model. Since we had sampled only a fraction of the $1,854 \times 1,854$ similarity matrix, the test data were insufficient for addressing how well the model could predict behaviorally measured similarity. To this end, we used online crowdsourcing to collect between two and three 
behavioral responses for each possible triplet of those 48 objects $(43,200$ choices $)$ and calculated choice probabilities for each pair of objects as a measure of their similarity. To estimate the noise in the fully-sampled matrix, we calculated the reliability by splitting behavioral data in half and generating two split-half similarity matrices. Then, we computed a predicted similarity matrix using our computational model and compared it to both the full similarity matrix and each split. The predicted and measured similarity matrices are depicted in Fig. $2 \mathrm{~b}$. Both matrices were highly correlated (Pearson $r=0.90, p<0.001$, randomization test, $95 \% \mathrm{Cl}$ : 0.88 0.91 ), with the fit of each half again approaching noise ceiling (first half: $r=0.87$, second half: $r$ $=0.88$, reliability: $r=0.91$ ), demonstrating that the model was able to accurately reproduce behaviorally measured similarity even with very sparsely sampled data. This result highlights that despite the large number of objects and the complexity of natural stimuli, most of the largescale representational structure of objects measured through human similarity judgments can be captured by a fairly low-dimensional embedding.

\section{Are the model's dimensions interpretable?}

The results so far establish that the model dimensions are reproducible, can be used to accurately generate similarities between pairs of objects, and predict individual behavior close to the noise ceiling. However, they leave open the degree to which individual model dimensions can be interpreted meaningfully. If the dimensions are interpretable, then the objects with the highest weight in a given dimension should share certain properties that are easy to identify. In Fig. 3, we illustrate the interpretability for a subset of dimensions by displaying the object images with the largest weights in those dimensions.

Visual inspection of the dimensions suggests they are interpretable and reflect conceptual and perceptual properties of those objects. Among others, the model identified dimensions that appear to reflect the semantic membership of those objects, such as dimensions related to food, animals, furniture, vehicles, or tools. In addition, a number of dimensions appear to reflect other conceptual properties, such as being metallic or hard, valuable, disgusting, heat-related or water-related. Finally, some dimensions appear to reflect perceptual properties, such as the roundness of objects, their elongation, flatness, color, shininess, or patterned texture. For later use throughout this manuscript, we assigned intuitive labels to each dimension (e.g. "animalrelated/organic", "colorful").

To explicitly test this interpretability in naïve observers, we asked 20 laboratory participants (15 female, 5 male) to provide labels for those dimensions, based on viewing objects sorted by their numeric value along each dimension. Since interpretability need not be limited to a single label, we visualized the naming results using word clouds, for which more frequently provided labels are displayed with a larger font. While participants' descriptions varied and tended to focus more on extreme examples of a dimension, they exhibited a remarkably close correspondence to the labels we had assigned to the dimensions (see Extended Data Figure 2 for naming of all 49 dimensions). 
Dimension 3: animal-related / organic
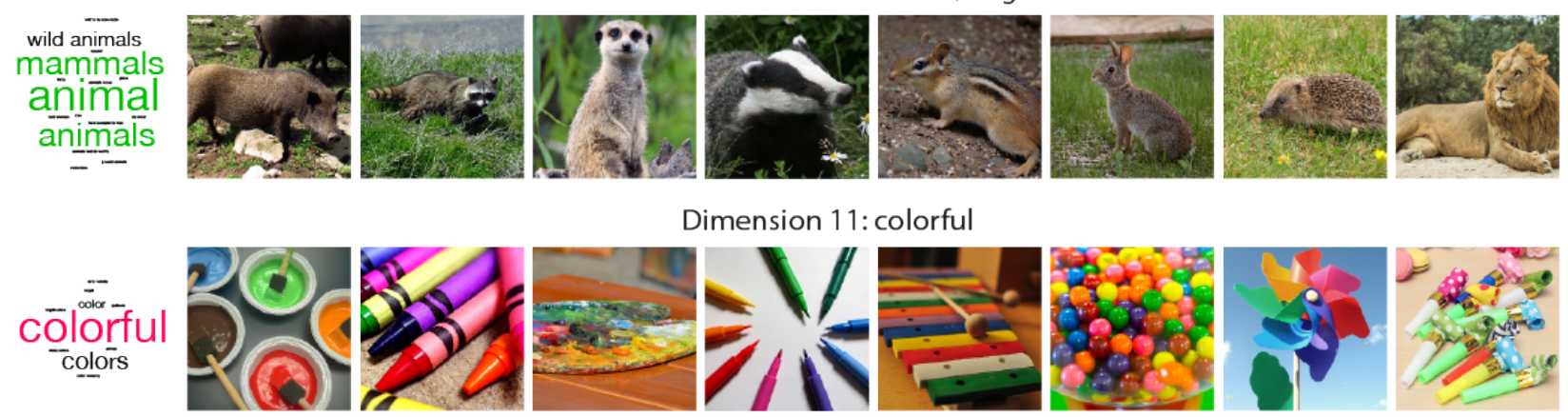

Dimension 12: valuable / special occasion-related
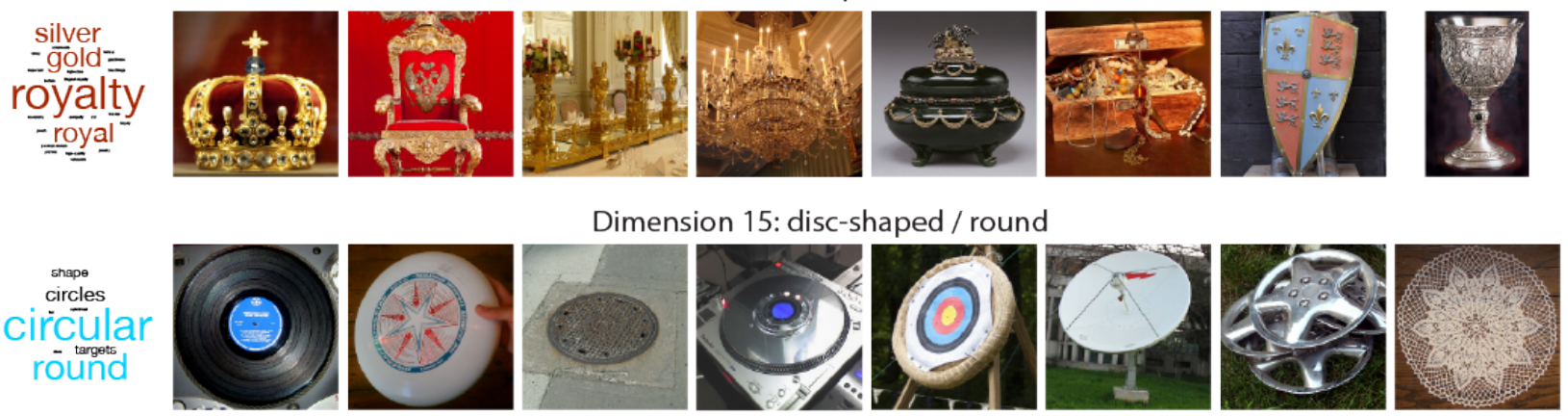

Dimension 17: many small things / coarse pattern
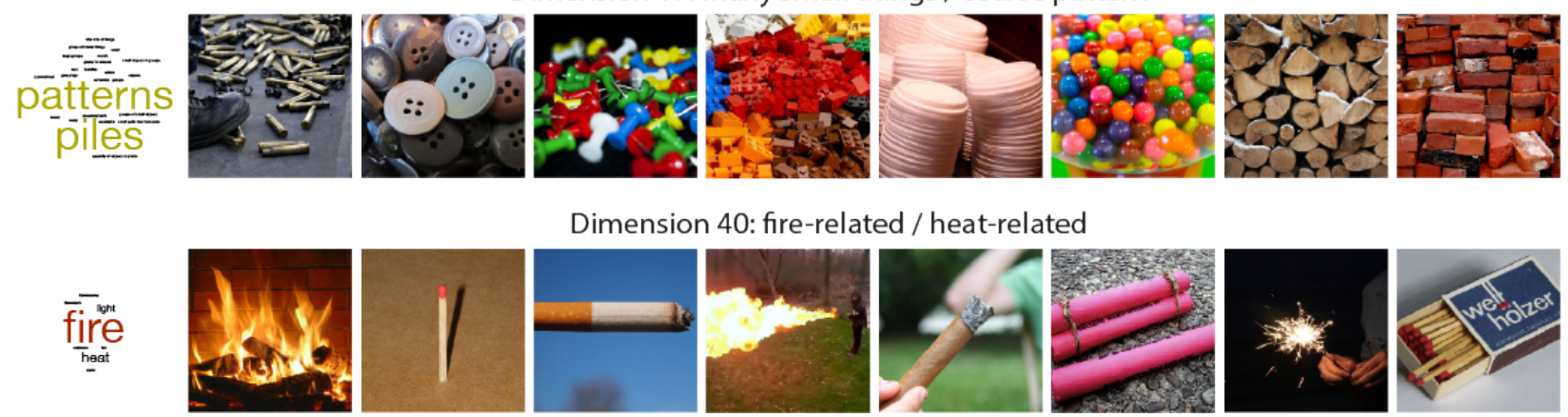

Dimension 40: fire-related / heat-related

Fig. 3 | Example object dimensions illustrating their interpretability. The images reflect the objects with the highest weights along those dimensions. Word clouds illustrate the labels provided by 20 participants to visual exposure of those dimensions, weighted by their naming frequency. While responses tended to focus more on extreme examples, they generally exhibited a close correspondence to the dimension labels we generated, which are shown above each set of images (see Extended Data Figure 2 for labels and word clouds of all dimensions).

Having established the interpretability of object dimensions, we can explore what dimensions a given object is composed of. For that purpose, in Fig. 4 we visualize a range of different objects using circular bar plots ("rose plots"), where the angle and color of a petal reflect the object dimension and the length of the petal reflects the degree to which the dimension is expressed in that object. For example, the image of noodles is characterized mostly by being food-related, repetitive and stringy. In contrast, the image of a rocket is characterized mostly by being transportation-related, flying-related, fire-related, artificial and shiny. This visualization demonstrates that some dimensions indeed reflect perceptual properties, since they are specific for the chosen object images: They may not show up for a different image of the same object 
and might have been missed completely if words had been chosen instead of images. In addition, the visualization demonstrates that objects are indeed characterized by a rather small number of dimensions (see below for a quantification).
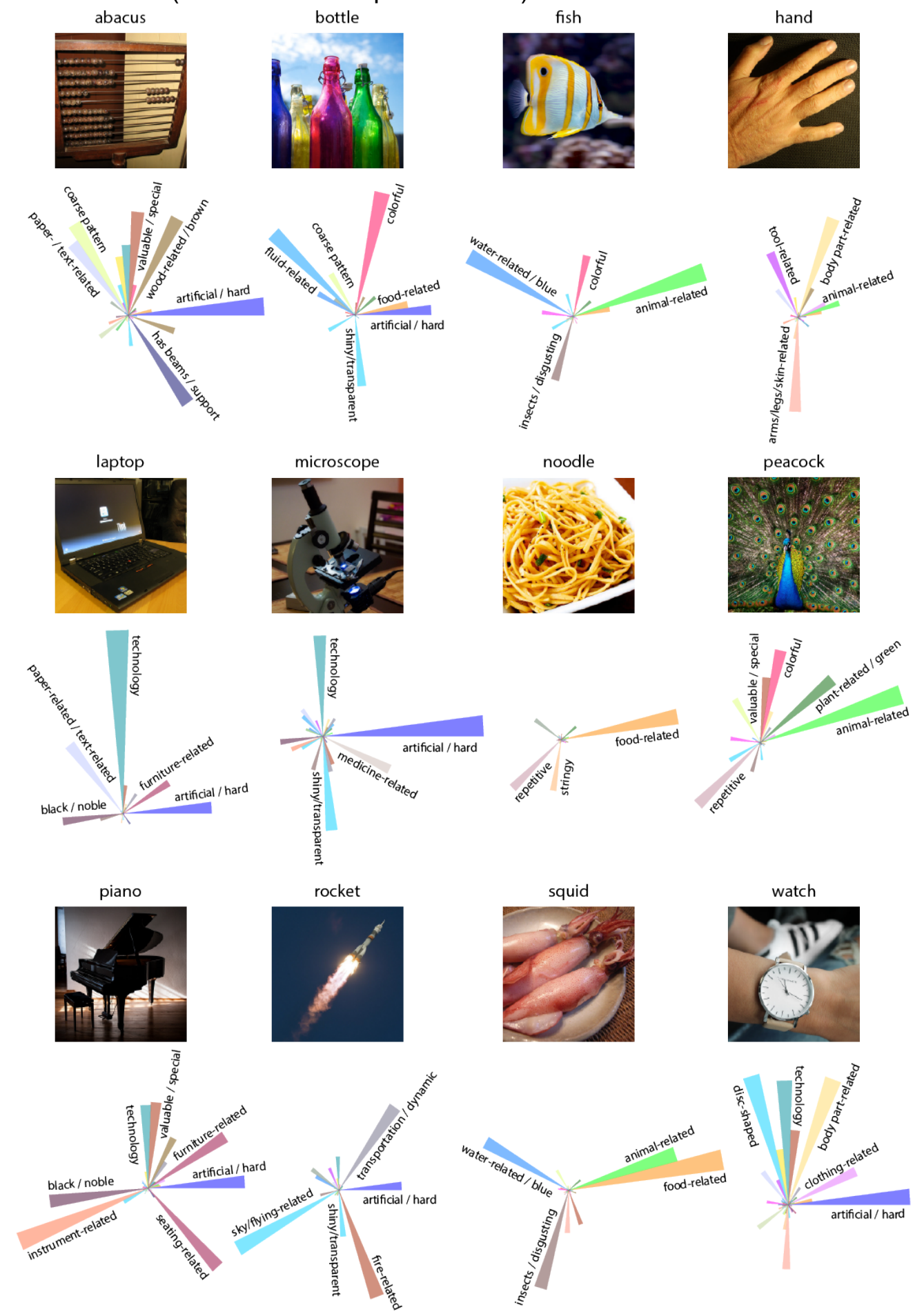

Fig. 4 | Illustration of example objects with their respective dimensions, using circular bar plots ("rose plots"). The length of each petal reflects the degree to which an object dimension is expressed for the image of a given object. For display purposes, dimensions with small weights are not labeled. 


\section{Natural object categories as emergent property of similarity embedding}

To characterize the relative similarity of objects to each other and explore the distribution of dimensions across objects, we combined two common visualization tools. First, we projected the 49-dimensional similarity embedding to 2 dimensions using $t$-distributed stochastic neighborhood embedding ( $t$-SNE, dual perplexity: 5 and 30), initialized using metric multidimensional scaling. This approach has been shown to preserve the global similarity structure while providing a higher degree of interpretability at the local similarity level than multidimensional scaling alone ${ }^{24}$. Second, in this two-dimensional plot we visualized each object using rose plots (as in Fig. 4).

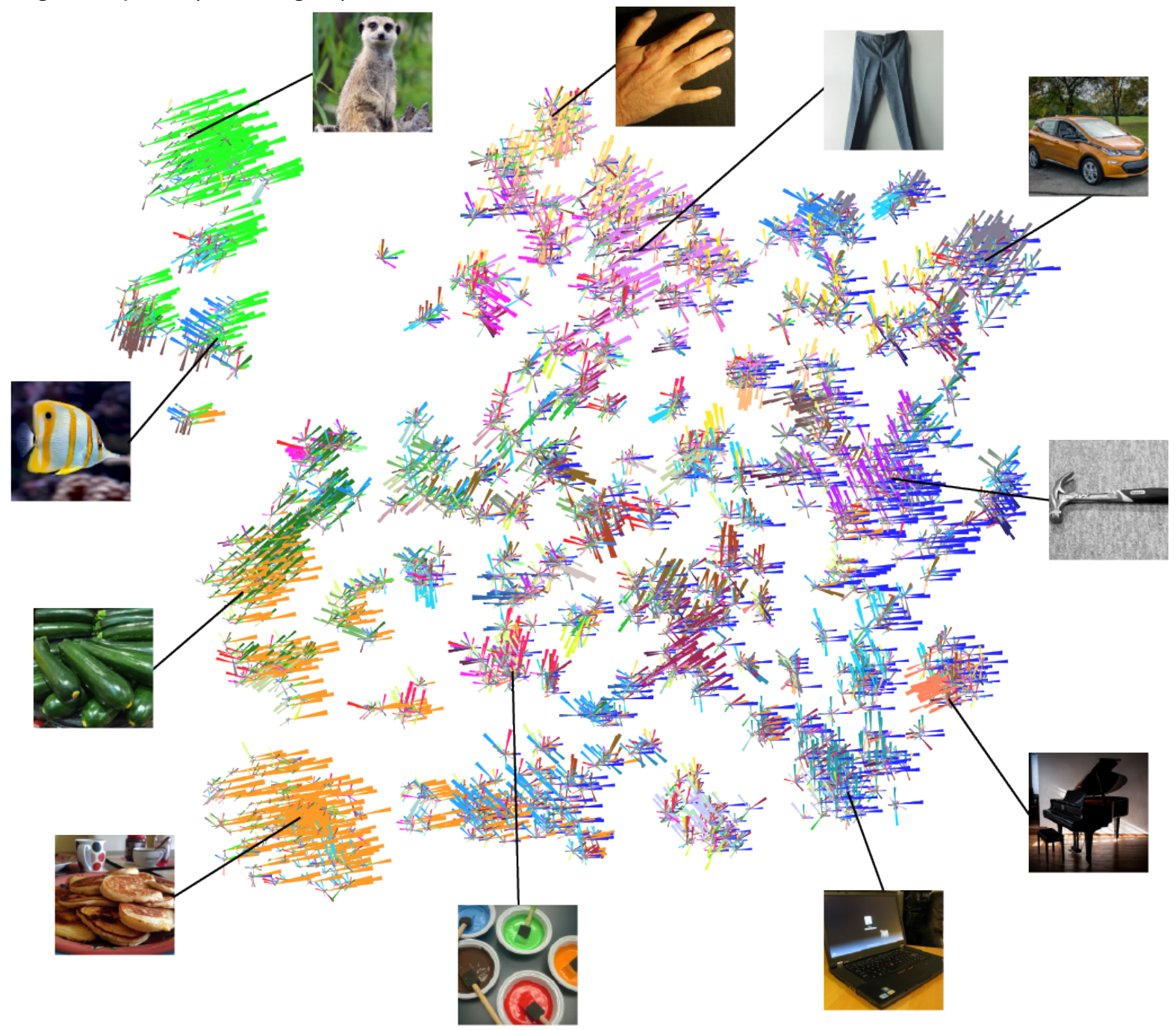

Fig. 5 | Two-dimensional visualization of the similarity embedding, combining dimensionality reduction (MDS-initialized $t$-SNE, dual perplexity: 5 and 30, 1,000 iterations) with rose plots for each object (see Fig. 4). At the global structure level, the results confirm the well-known distinction between "animate inanimate" or "man-made - natural" objects, with some exceptions (see main text). In addition, the different clusters seem to reflect broader object categories which emerge naturally from object similarity judgments. However, dimensions are not restricted to those clusters but are expressed to different degrees throughout this representational space. 
The resulting visualization (Fig. 5) reveals several interesting features of the similarity embedding. The global similarity structure seems to highlight the well-known distinctions between "animate - inanimate" and "natural - man-made", but also reveals three differences. First, representations of humans and human body parts are largely separate from animals and closer to the man-made objects, in line with neuropsychological findings ${ }^{11,25}$ and demonstrating an important limitation of simply applying taxonomic relationships for studying mental representations ${ }^{26}$. Second, processed food was found to be more closely related to living and natural objects, acting as an exception to the universality of naturalness as a critical dimension of object representations, again in line with patient data ${ }^{27}$. Third, the weights of dimensions do not reflect binary membership to the categories of e.g. "natural - man-made" objects. For example, focusing on the dimension "artificial / hard" (dark blue, rightward-oriented bars), this dimension was most strongly expressed on the right of the graph but became weaker when moving to the left, towards animals, food, and natural objects.

In addition to this global structure, many objects formed clusters related to high-level categories (e.g. animals, tools, vehicles, musical instruments). This indicates that categorization behavior for many categories may be accounted for by the similarity of objects, a property which has been discussed previously ${ }^{28,29}$ but which had not been tested on a large set of natural objects. To test how well natural categories could be predicted by the similarity embedding, we used 18 unique high-level categories identified in the THINGS database ${ }^{17}$ and used a cross-validated nearest centroid classifier to predict category membership for each of the 1,112 objects of those categories. The classifier performed at $86.42 \%$ (chance performance: $5.56 \%$ ), on par with a recent semantic embedding of object meaning ${ }^{30}(85.97 \%)$ that had been trained on billions of words, demonstrating that the dimensions we identified allow the prediction of categorization behavior for a large number of natural categories.

Finally, the visualization reveals that certain combinations of dimensions are critical for forming different types of categories. Indeed, many subcategories can be explained by defining features: objects with large weights in "animal-related" and "water-related" dimensions are likely sea creatures, while objects with large weights in "plant-related" and "food-related" dimensions are likely vegetables. For example, an abacus can be explained as a combination of several dimensions, such as "artificial / hard", "wood-related", "valuable / special", and "coarse pattern".

\section{How many dimensions for an object?}

The visualizations in Figs. 4 and 5 suggest that some objects are easy to characterize with a relatively small number of dimensions. This indicates that, while all 49 dimensions are useful for some objects, individual odd-one-out judgments of objects may be predicted accurately with a smaller number of dimensions. Since the model performs close to the noise ceiling at predicting behavior, we should be able to produce a lower bound estimate for the number of relevant object dimensions for characterizing individual behavior and the global similarity structure. To this end, we carried out a dimension elimination approach. The reasoning behind this approach is that if a dimension does not matter for behavior, then setting it to 0 should not affect predictive 
performance of the model. Therefore, for each object, we set the dimension with the lowest weight to 0 , predicted behavior in the test set, recomputed the similarity matrix, and compared how this affected the predictive performance of the model. We then repeated this elimination process until only one dimension was left. Importantly, this procedure eliminates not entire dimensions from all objects, but eliminates different dimensions for each object. If behavior is driven by a larger number of dimensions than retained, this would be reflected in reduced model performance. The results of these analyses demonstrate that in order to achieve $95-99 \%$ of the performance of the full model in explaining behavioral judgments in the odd-one-out task, a total of 6 to 11 dimensions are required (Fig. 6a), and to explain $95-99 \%$ of the variance in the similarity matrix, a total of 9 to 15 dimensions are required (Fig. $6 \mathrm{~b}$ ). Thus, while the representational space of objects can be captured by a comparably low-dimensional embedding, for judging the similarity of objects, on average humans indeed seem to integrate across a larger number of those dimensions.

a

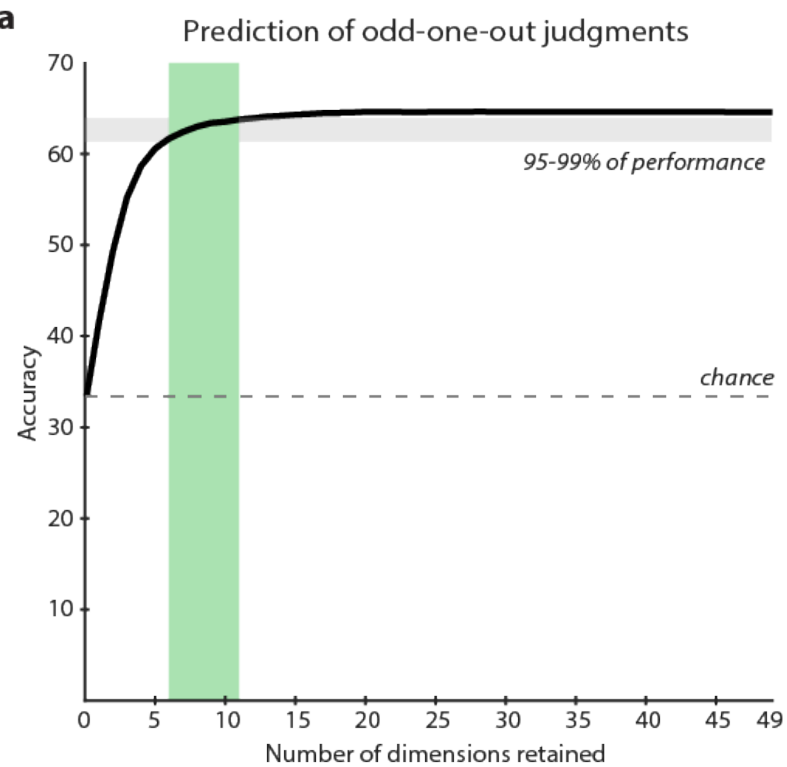

b

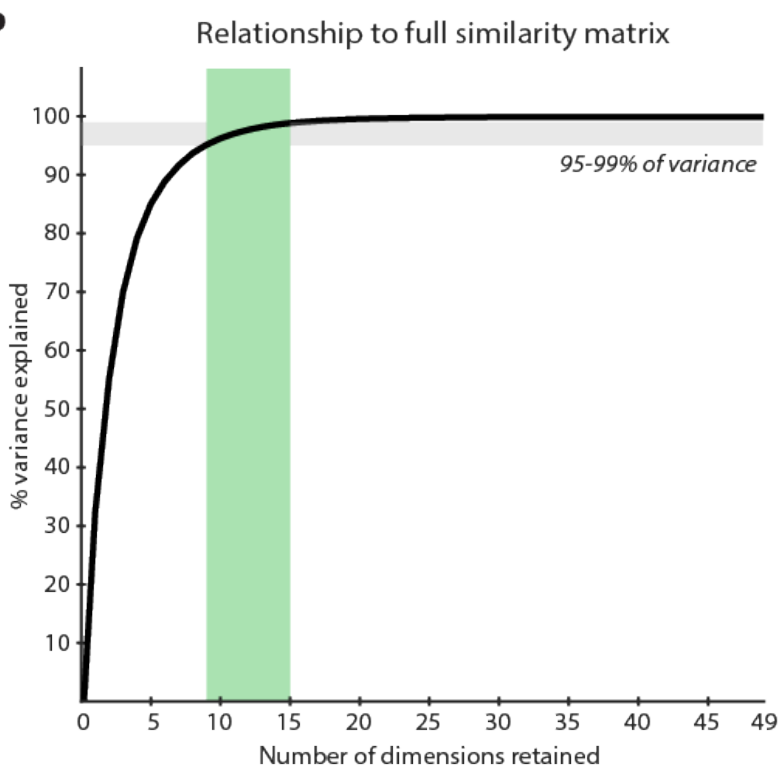

Fig. 6 | How many dimensions are required to capture behavioral judgments and object similarity? By iteratively setting the dimensions with the smallest numeric value to 0 , we estimated the effect of eliminating those dimensions from judgments. A drop in model performance indicates behavioral relevance of those dimensions. For explaining 95 to $99 \%$ of the predictive performance in behavior, between 6 and 11 dimensions are required, while for explaining 95 to $99 \%$ of the variance in similarity, between 9 and 15 dimensions are required.

\section{Typicality as emergent property of similarity embedding}

While objects are characterized by several different dimensions, it is unclear to what degree these dimensions merely reflect binary properties of the objects (e.g. "is an animal", "is a tool", "is a vehicle") or rather the degree to which they are present in an object (e.g. "animacy", "manipulability", "utility for transportation"). While we were able to predict the high-level category of objects from the embedding, we did not test whether the continuous nature of the dimensions was informative about the degree to which a dimension is expressed in an object. The 
continuous nature of dimensions may be reflected in the typicality of objects within their corresponding category. Interestingly, this would demonstrate that not only object categories, but also typicality can be described as an emergent property of object similarity. To test whether the numeric value of a dimension reflected typicality, we used online crowdsourcing to collect typicality ratings for words of the 27 high-level categories in the THINGS database. Of those categories, 17 could be related to dimensions of our embedding according to their dimension labels, and consequently, we tested their correspondence with typicality. The results of these analyses are shown in Fig. 7 and Extended Data Table 1. Despite the typicality ratings being based on words and the dimensions on images, 14 out of 17 dimensions revealed a significant positive relationship with typicality scores (Spearman's $\rho$ : 0.26-0.62, all $p<0.05$, one-sided, FDR-corrected for multiple comparisons). These results demonstrate that typicality may indeed be an emergent property of the object dimensions. However, the results also reveal that some dimensions with a weaker relationship do not seem to reflect a purely category-related semantic code but may incorporate other, perhaps perceptual aspects.

Dimension values vs. typicality ratings
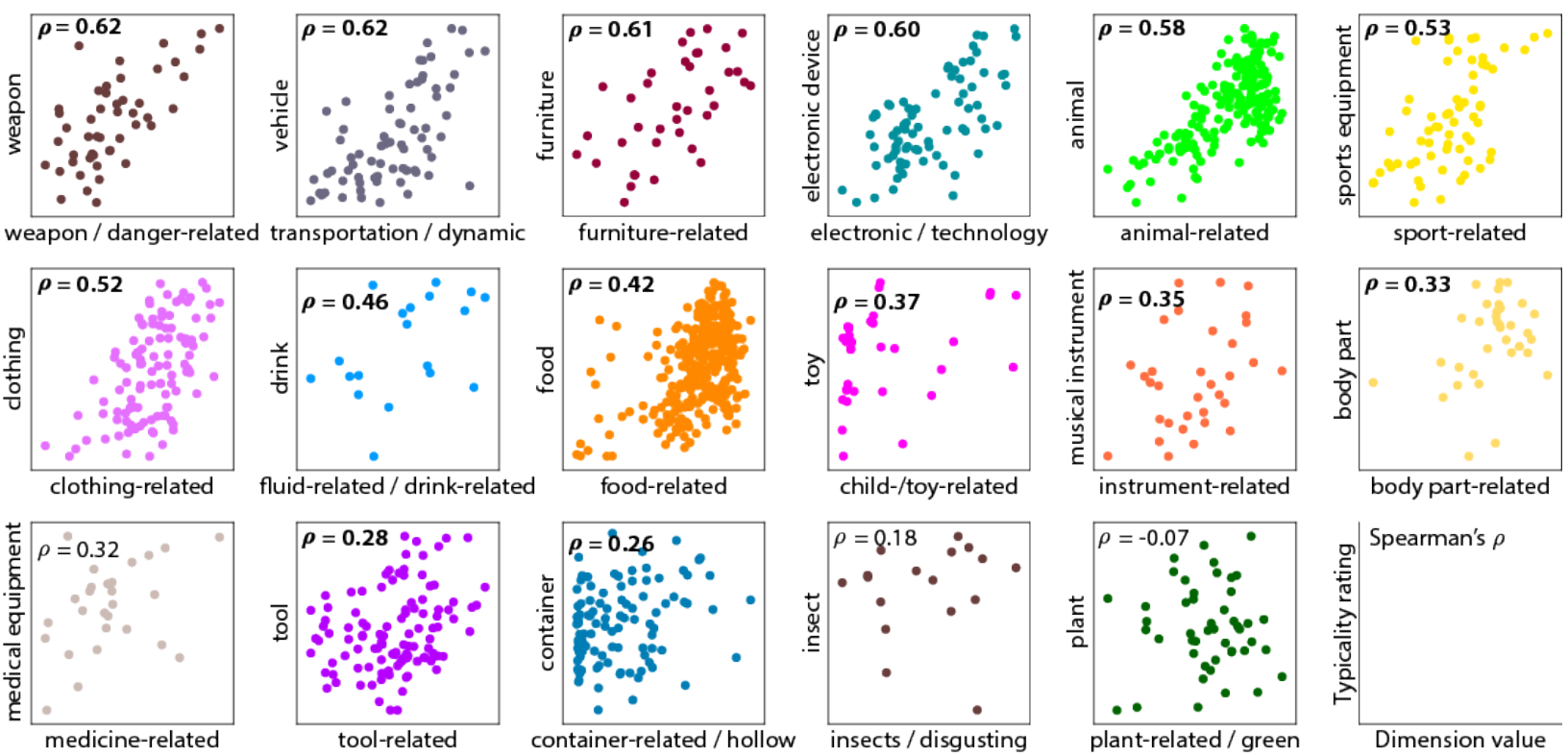

Fig. 7 | The relationship between seemingly categorical dimensions and typicality ratings of those categories. Many of these dimensions exhibited a positive relationship between the numeric value of objects along that dimension and the typicality of category membership. This demonstrates that even seemingly categorical dimensions reflect the graded nature of the underlying dimensions and that typicality may be an emergent property of those dimensions. All results were min-max scaled for better comparability. Significant relationships between both variables are displayed in bold typeface $(p<0.05$ one-sided, FDR-corrected for multiple comparisons). See Extended Data Table 1 for individual inferential statistical results.

\section{Human ratings along model dimensions allow generating similarity scores for arbitrary object images}


A generative model of object similarity would open the possibility to directly operate on the dimensions rather than having to collect similarity judgments. To what degree can the representational embedding identified from the odd-one-out judgments act as a generative model of object similarity? A simple way to test this idea is to ask participants to provide direct ratings of objects along the dimensions of the embedding ${ }^{31}$. If it is possible to generate similarity from human ratings of dimensions, this would also serve as a stricter test of their interpretability.

For a set of 20 object images selected at random from the 1,854 objects, we asked 20 laboratory participants ( 15 female, 5 male) to rate those objects along the 49 object dimensions of the representational embedding (Fig. 8a). Rather than providing them with semantic labels for the dimensions, participants were shown example images along a continuous rating scale and were asked where along that scale they would place those objects. In the next step, responses for each dimension were averaged across participants and used in place of the model dimensions to generate a human-predicted similarity matrix. Importantly, since the focus here was to provide proof-of-concept for the usefulness of dimensions, participants had not been trained on this task.

The comparison of similarity from direct dimension ratings and the reference similarity matrix from the model embedding (Fig. 8b) revealed a generally close correspondence between the two matrices (Pearson $r=0.85, p<0.001$, randomization test, $95 \% \mathrm{Cl}: 0.80-0.89$ ). This result demonstrates that humans are able to judge dimensions for objects to allow for a good reconstruction of similarity from dimension ratings. Importantly, since this task was carried out with participants that were not trained on the use of the rating scale and were not instructed regarding the interpretation of the dimensions, this result underscores the interpretability and usefulness of the dimensions. 

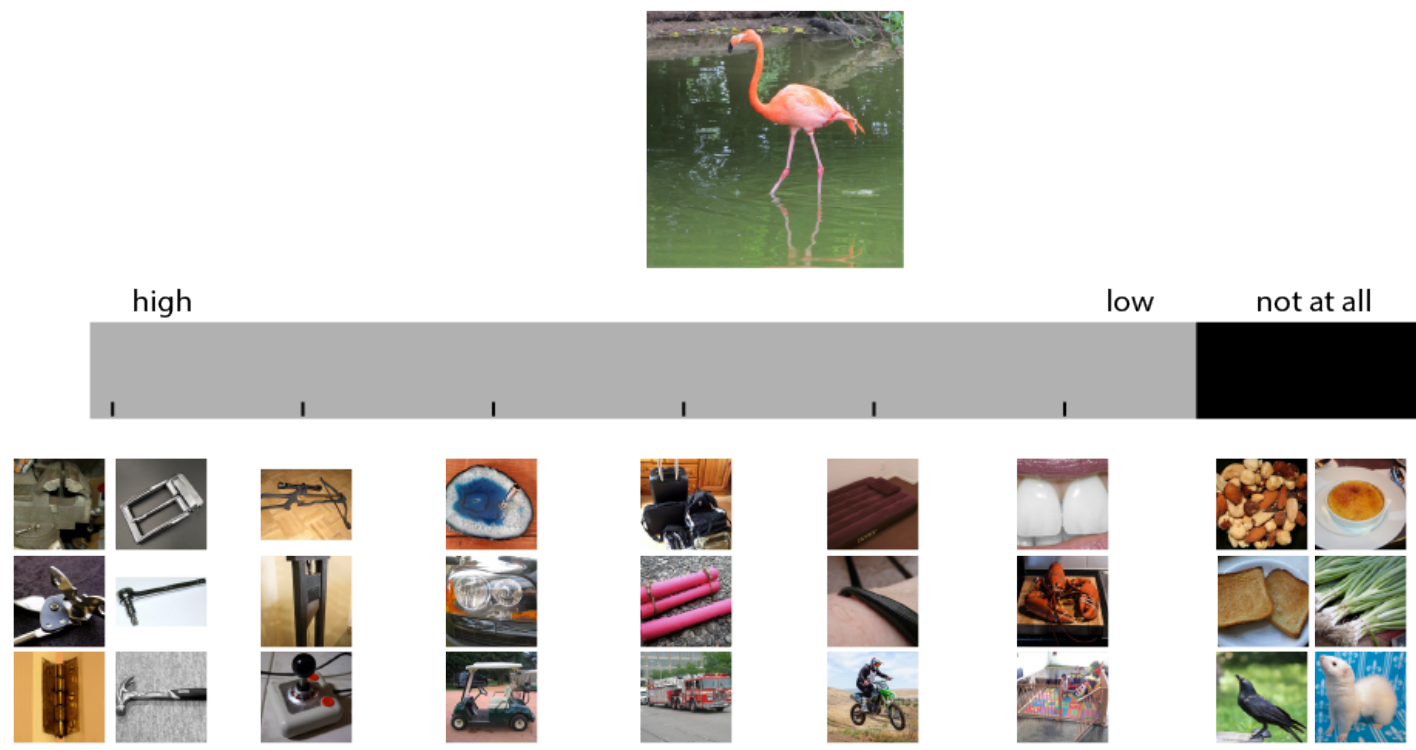

b

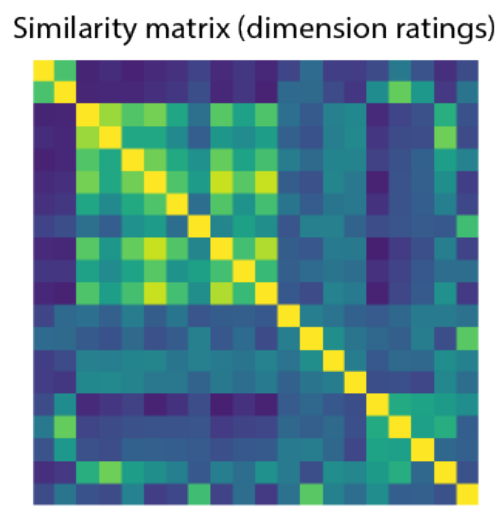

20 randomly sampled objects
Similarity matrix (reference)

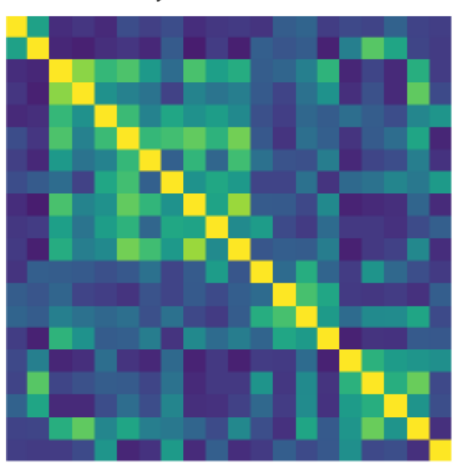

0

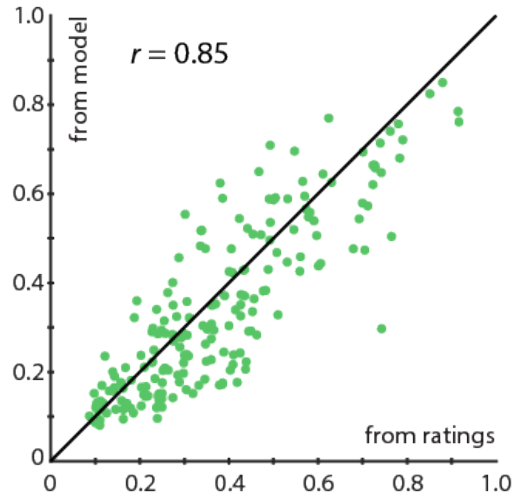

Fig. 8 | Task and results of direct ratings of dimensions. a 20 participants were asked to indicate with a mouse click where they believed objects would fall along all 49 model dimensions. Rather than providing participants with dimension labels, the rating scale was spanned by example images along the currently rated dimension (in this example, dimension 1, "artificial/hard"). b Results for the 20 tested objects revealed a good reconstruction of object similarity by dimension ratings when comparing it to the similarity predicted from the embedding that served as a reference (Pearson $r=0.85, p<0.001$, randomization test, $95 \% \mathrm{Cl}: 0.80-0.89$ ). These results further support the idea that dimensions are interpretable and that they can be used to directly generate object similarities.

\section{Discussion}

Identifying the structure of our internal mental representations is a central goal in the cognitive sciences. For the domain of natural objects, this may seem particularly challenging, given the high complexity of our visual world that contains thousands of objects with a seemingly countless number of possible object properties. Here, using a triplet odd-one-out task on a wide 
range of object images, we demonstrated that it is possible to characterize the similarity structure and individual human behavioral judgments with a low-dimensional representational embedding learned directly from human choice behavior. The model revealed 49 meaningful object dimensions, each being interpretable with respect to the perceptual and conceptual properties of those objects, reflecting both basic perceptual properties of shape, color, texture as well as more high level properties such as taxonomic membership, function, or value. The embedding allowed the prediction of other forms of behavior, including high-level categorization and typicality judgments. By demonstrating that participants can use these dimensions to generate object similarity scores, these results open the avenue towards a generative model of object similarity judgments. Importantly, the resulting large-scale similarity matrix based on our representational embedding can act as a basis for testing formal computational models of categorization and category learning in the domain of natural objects ${ }^{31}$.

Being able to characterize mental representations of objects with a low-dimensional embedding is surprising, given their high degree of perceptual variability and our broad semantic knowledge of them ${ }^{32}$. Indeed, popular semantic feature production norms ${ }^{15,16}$ have revealed thousands of binary features that participants name when asked about their explicit knowledge of objects. Rather than attempting to capture all details of our semantic knowledge of objects with binary properties, our results demonstrate that it is possible to achieve high predictive performance using only a small number of interpretable, continuous dimensions. It may be possible to generate these binary properties from the continuous dimensions in our model ${ }^{33}$, which would demonstrate that the implicit judgments in the odd-one-out task capture much of the explicit semantic knowledge of objects, but a general test of this idea would require the creation of feature production norms for the 1,854 objects used in the creation of the embedding. However, even if such norms were created, there are two reasons that their predictions may be limited. First, the dimensions revealed in this work are focused around the properties most relevant for discriminating among different objects, while feature production norms would likely contain much more - often idiosyncratic - information than required for those distinctions. Second, when using object words and an explicit feature naming task, participants often omit critical features $^{14,34}$. By using object images and an implicit, non-verbal task, it is possible to capture perceptual dimensions of objects with a representational embedding that might otherwise be missed.

In contrast to traditional data-driven approaches that identify multidimensional feature spaces using dimensionality reduction techniques such as multidimensional scaling ${ }^{35,36}$, factor analysis $^{37,38}$, or additive clustering ${ }^{39}$, for the present model we made two assumptions that support the interpretability of dimensions, motivated by the observation of how objects are typically characterized ${ }^{21,22}$ : (1) dimensions are sparse, i.e. each object carries only some dimensions but not others, and (2) dimensions are positive, i.e. each object is characterized by a combination of dimensions that are present to a certain degree and that add up without canceling each other out. By incorporating these assumptions, our model not only yields interpretable dimensions, but also reflects a blend between two common model families used to characterize objects: dimensional models that assume continuous dimensions, and featural models that assume the presence and absence of, mostly binary, object properties ${ }^{9,40}$. Analyses 
of category-related typicality judgments demonstrate that the continuous nature of the dimensions is informative as to the degree to which these dimensions are expressed in objects, demonstrating that continuous dimensions allow us to generalize beyond binary categorical assignment of semantic attributes (e.g. "is animate") ${ }^{41}$. In addition, while traditional pairwise assessment of similarity typically neglects the importance of object context ${ }^{3,42}$, by using a triplet odd-one-out task this type of embedding in principle allows generating object similarity for arbitrary contexts imposed by focusing on a chosen subset of objects (e.g. animals). The degree to which the embedding carries such fine-grained information will need to be tested in future studies.

While mathematically, there are an infinite number of possible ways in which object representations can be characterized by a set of dimensions, identifying a broad range of meaningful and predictive dimensions with a bottom-up, data-driven model offers a systematic approach for the identification of meaningful dimensions, complementing traditional top-down, theory-driven approaches. Ultimately, however, further studies are required to validate the specificity of different dimensions in this model and link them to representations in the human brain. One intriguing prediction of our model is that specific deficits in recognizing objects found in patients with focal lesions may be tied more to specific dimensions identified in this study or regions in representational space than to specific object categories ${ }^{25,27}$. For example, given the prominence of the dimension "shiny / transparent", one might expect to find specific deficits associated with surface materials of objects. Likewise, based on the representational proximity of clothing and body parts, one might expect a deficit in one to be associated with a deficit with the other.

While we demonstrated that most dimensions were highly reproducible across different random initializations of the model, using a smaller subset of the data for building the embedding revealed a smaller number of dimensions (results not shown), indicating that the dimensionality of the embedding is a function on the amount of data used. Further, while the sparsity constraint is an important feature of the model, one limitation is that it may lead to very similar dimensions being merged (e.g. "plant-related and green"). Ultimately, additional data will be required to test the degree to which these dimensions remain stable or whether further dimensions will appear. However, since the model performed close to noise ceiling at predicting similarity judgments and yielded interpretable dimensions, this demonstrates that the representational embedding already provides a useful description of behavioral judgments and object similarity.

Finally, the prediction of similarity from direct ratings of dimensions was based on 20 objects that were part of creating the original embedding, which may slightly overestimate the ability to generate similarity from dimension ratings. However, given the large number of objects used in this study, we believe it to be unlikely that those $\sim 1 \%$ of the objects would strongly bias the results, and collecting ratings from a different set of objects would have required generating, characterizing and testing a different model, which was prohibitive in the context of this study. Future studies with the goal of training participants to generate dimension ratings could rely on a separate set of objects for relating predicted with measured similarity. 
The approach proposed in this study opens the avenue for many related questions: To what degree are the dimensions shared between different individuals ${ }^{43,44}$, and how are they affected by gender, age, culture, education, other sociodemographic factors, and individual familiarity with the objects? To what extent do the representations depend on the exact task, and can other similarity tasks evoke similarly fine-grained representations ${ }^{45,46}$ ? What are the representational dimensions in other domains, such as words, faces, places, or actions? Finally, what makes those representations similar to those found in deep convolutional neural network models of vision ${ }^{47}$, semantic embeddings learned on word co-occurrence statistics in large text corpora $^{22,30,48}$, or brain activity in humans ${ }^{49-53}$ ? Addressing these questions will be important for a comprehensive understanding of mental representations of objects across people and different domains.

\section{Methods}

\section{Participants}

A total of 5,983 workers from the online crowdsourcing platform Amazon Mechanical Turk participated in the triplet odd-one-out experiments, which consisted of the creation of the fullysampled matrix of 48 objects (121 workers, after exclusion 100; 46 female, 54 male), the data for training and testing the computational model (5,526 workers, after exclusion 5,$301 ; 3,159$ female, 2,092 male, 19 other, 31 not reported), and the 1,000 randomly chosen triplets used for the estimation of a noise ceiling (336 workers, after exclusion 325; 156 female, 103 male, 66 not reported). In addition, a total of 337 workers (no exclusions; 198 female, 131 male, 8 not reported) participated in the creation of the typicality norms. All workers were located in the United States, and worker age was not assessed. For the odd-one-out task, workers were excluded if they exhibited overly fast responses in at least 5 sets of 20 trials (speed cutoff: $25 \%$ or more responses $<800 \mathrm{~ms}$ and $50 \%$ or more responses $<1100 \mathrm{~ms}$ ) or if they carried out at least 200 trials and showed overly deterministic responses (> 40\% of responses in one of the three odd-one-out positions, expected value: 33\%). All workers provided informed consent. The online research was approved by the Office of Human Research Subject Protection (OHSRP) and conducted following all relevant ethical regulations, and workers were compensated financially for their time.

In addition, 20 laboratory participants (15 female, 5 male, mean age: 26.25 , std: 6.39 , range 19$41)$ took part in the dimension labeling and the dimension rating experiment. All laboratory participants provided written informed consent and were compensated financially for their time. The laboratory experiments were carried out following all relevant ethical regulations and rules of the National Institutes of Health (NIH) Institutional Review Board (NCT00001360).

\section{Object images and odd-one-out task procedure}

The 1,854 images of objects used in this study were the reference images that had been used previously for validating the concepts of the THINGS database ${ }^{17}$. The images depict objects 
embedded in a natural background and were all cropped to square size, with the exception of a small number of images that didn't fit into a square and that were padded with white background on both sides. Importantly, the validation task of the THINGS database demonstrated that the objects in the 1,854 images were generally nameable, i.e. it can be assumed that most participants were sufficiently familiar with the objects to be able to name them. The triplet oddone-out task was carried out in sets of 20 trials, and workers could choose how many sets they would like to carry out. On each trial, workers were shown three object images side by side in a browser window and were asked to report the image that was the least similar to the other two. Workers were told that they should focus their judgment on the object, but to minimize bias they were not given additional constraints as to the strategy they should use. In addition, participants were instructed that in case they did not recognize the object, they should base their judgment on their best guess of what the object could be. Participants responded with a mouse click on the respective image, which initiated the next trial after an intertrial interval of $500 \mathrm{~ms}$. Each object triplet and the order of stimuli was chosen randomly, but in a way that after data collection each cell in the $1,854 \times 1,854$ similarity matrix had been sampled at least once.

To yield a diverse set of objects for the fully sampled similarity reference dataset, the 48 objects were chosen by carrying out spectral clustering on publicly-available 300-dimensional sense vectors of all 1,854 objects $^{30}$ with 48 clusters and by choosing one object per cluster randomly.

\section{Details of computational modeling procedure}

The model and preliminary results were presented previously at a conference ${ }^{33}$. The model was implemented as a computational graph in TensorFlow (Version 1$)^{54}$. Each triplet was encoded using three one-hot vectors (length: 1,854), and each vector was linked to 90 latent dimensions, but with weights replicated across all three vectors. The 1,854 × 90 weights were initialized randomly (range $0-1$ ). Note that initializing the model with 200 dimensions led to very similar model performance and final number of dimensions (results not shown). The dot product was chosen as a basis for proximity for computational reasons, but using Euclidean distance led to very similar results (results not shown). The objective of the model optimization consisted of the cross-entropy, which was the logarithm of the softmax function, and a regularization term based on the L-1 norm to encourage sparsity,

$$
\sum^{n} \log \left(\frac{\exp \left(x_{i} x_{j}\right)}{\exp \left(x_{i} x_{j}\right)+\exp \left(x_{i} x_{k}\right)+\exp \left(x_{j} x_{k}\right)}\right)+\lambda \sum^{m}\|x\|_{1}
$$

where $x$ corresponds to an object vector, $i, j$, and $k$ to the indices of the current triplet, $n$ the number of triplets, and $m$ the number of objects. The regularization parameter $\lambda$ that controls the trade-off between sparsity and model performance was determined using cross-validation on the training set. In addition to sparsity, the optimization was constrained by strictly enforcing weights in the embedding $X$ to be positive. Minimization of this objective was carried out using stochastic gradient descent as implemented in the Adam algorithm ${ }^{55}$ using default parameters and a minibatch size of 100 triplets. After optimization was complete, dimensions for which weights of all objects were smaller than 0.1 were removed, leaving us with 49 dimensions. Empirically, the largest maximum weight of all excluded dimensions was 0.03 , while the 
smallest maximum weight of all included dimensions was 1.38. The dimensions were sorted in descending order by the sum of their weights across objects.

\section{Computation of similarity matrix from embedding}

We defined object similarity in the triplet odd-one-out task as the probability $\mathrm{p}(i, j)$ of participants choosing objects $i$ and $j$ to belong together, irrespective of context. Therefore, to compute similarity from the learned embedding for all 1,854 objects, we created all predicted choices for all possible $\sim 1.06$ billion triplets and calculated the mean choice probability for each pair of objects. For the fully-sampled similarity matrix of 48 objects used for testing the performance of the model at predicting object similarity (Fig. 2), we created a different similarity matrix that was constrained only by this subset of 48 objects.

\section{Reproducibility of embedding dimensions}

Due to the stochasticity of the optimization algorithm, each time the model is re-run, we will likely end up with a different set of dimensions. To determine the stability of each dimension in our 49-dimensional embedding, we re-ran the model 20 times, each time with a different random initialization. Next, we correlated each of the 49 original dimensions with all dimensions of one of the 20 reference embeddings and chose the best-fitting dimension across all correlations as the closest match. Then, we applied a Fisher z-transform to the correlations, averaged them across all 20 reference embeddings and inverted the Fisher z-transform to get a mean reliability for each dimension across all 20 embeddings. While the resulting comparison may exhibit a slightly positive bias due to choosing the best fit, a split-half cross-validation between all objects demonstrates nearly indistinguishable results (maximum difference $r=$ 0.01).

\section{Category prediction}

The categorization performance of the representational embedding was tested on 18 of the 27 categories in the THINGS database. Objects that were members of multiple categories were removed. Of the 9 categories that were removed, 7 were subcategories of other categories (e.g. "vegetable" in "food") or had fewer than 10 objects after removal of non-unique objects. The remaining 18 categories were as follows: animal, body part, clothing, container, electronic device, food, furniture, home decor, medical equipment, musical instrument, office supply, part of car, plant, sports equipment, tool, toy, vehicle, and weapon. These categories comprised 1,112 objects. Classification was carried out using leave-one-object-out cross-validation. For training, Centroids for all 18 categories were computed by averaging the 49-dimensional vectors of all objects in each category, excluding the left-out object. The membership of this remaining object was then predicted by the smallest Euclidean distance to each centroid. This procedure was repeated for all 1,112 objects, and prediction accuracy was averaged. For the corresponding analysis with a semantic embedding, we used publicly-available 300-dimensional sense vectors ${ }^{30}$. 


\section{Typicality ratings}

Typicality ratings were collected for all 27 object categories in the THINGS database, with the goal of including them as metadata for the database. However, for the purpose of this study, we focused on the 17 categories for which the labels indicated a relationship of dimensions with specific object categories. Typicality ratings were collected by asking workers to rate the typicality of an object as belonging to a given category, using a Likert scale from 0 to 10 . Each rating was collected 40 times. To improve comparability of the use of the Likert scale, each workers' responses were z-scored before they were merged with other responses.

\section{Dimension naming task and construction of word clouds}

In the dimension naming task, laboratory participants were asked to provide labels for different dimensions after inspecting them. This was achieved by showing them example object images along a continuous scale for a given dimension, comparable to the display in Fig. 8a. Participants could further inspect dimensions by clicking on example objects, which would reveal more object images in this range. The exact object images shown varied between dimensions. Participants were instructed not to focus exclusively on the top of the scale but to take the entire distribution of objects into account. After having studied a dimension, they were allowed to provide up to three verbal labels for the dimension. Word clouds displaying participants' responses according to the frequency of provided object labels were constructed using the function wordcloud in MATLAB (Mathworks, Natick, MA), using default parameters.

\section{Object dimension rating task}

The object dimension rating task was carried out after the dimension naming task. Participants were shown a reference image on the top and were asked to rate where they would place the object along a Likert scale, according to the meaning of the dimensions explored previously (Fig. 8a). Participants were recommended to take two of the 7 shown levels (demarcated by the object images), and for the given object judge if it was better characterized by one or the other level. The rating task was carried out for all 49 dimensions sequentially, on images of the following 20 objects chosen randomly from the set of 1,854 objects: bazooka, bib, crowbar, crumb, flamingo, handbrake, hearse, keyhole, palm tree, scallion, sleeping bag, spider web, splinter, staple gun, suitcase, syringe, tennis ball, woman, workbench, and wreck. Since many dimensions were at or close to 0 , the scale included a short range with all zero values ("not at all"). Further, to improve the discriminatory power of the scale, dimension values were converted to percentiles, with all percentiles lower than $20 \%$ set to 0.2 . After ratings had been collected, percentiles were converted back to their respective continuous values along the dimensions and averaged across participants. Further, for better comparability to the original dimensions, dimension values were scaled in a way that their minimum rating corresponded to 0 . Then, the object dimensions were treated as new embedding dimensions for the 20 objects, and object similarity was calculated for them according to the procedure described above. Finally, the similarity matrix generated from the object ratings was compared to the similarity matrix from the full model. 


\section{Statistical analyses and confidence intervals}

Unless indicated otherwise in the text, statistical analyses were conducted using classical parametric statistical tests and, when required, corrected for multiple comparisons using false discovery rate (FDR). Data distribution was assumed to be normal but this was not formally tested. All tests were two-tailed unless denoted otherwise. Non-parametric randomization tests on correlations between predicted and measured similarity matrices were conducted by creating 100,000 similarity matrices based on randomly shuffling object labels, re-running the correlation with the measured similarity matrix, and calculating $p$-values as the percentage of permutations reaching or exceeding the true similarity. Error bars reflect $95 \%$ confidence intervals and were created based on the standard error of the mean or - when no distribution was available - based on the standard deviation of 1,000 bootstrap samples.

\section{Data availability}

The learned embedding, triplet odd-one-out behavioral data for testing model performance, typicality scores, participant-generated dimension labels, and dimension ratings are available under https://osf.io/z2784. The behavioral data used for training the model are available from the corresponding author upon request.

\section{Code availability}

To reproduce relevant analyses and figures, relevant Matlab scripts and functions are available under https://osf.io/z2784. The computational modeling code to create an embedding is available from the corresponding author upon request.

\section{References}

1. Biederman, I. Recognition-by-components: A theory of human image understanding.

Psychol. Rev. 94, 115-147 (1987).

2. Edelman, S. Representation is representation of similarities. Behav. Brain Sci. 21, 449-467 (1998).

3. Nosofsky, R. M. Attention, similarity, and the identification-categorization relationship. J. Exp. Psychol. Gen. 115, 39-57 (1986).

4. Goldstone, R. L. The role of similarity in categorization: Providing a groundwork. Cognition 52, 125-157 (1994). 
5. Rosch, E., Mervis, C. B., Gray, W. D., Johnson, D. M. \& Boyes-Braem, P. Basic objects in natural categories. Cognit. Psychol. 8, 382-439 (1976).

6. Hahn, U. \& Chater, N. Concepts and similarity. in Knowledge, concepts and categories (eds. Lamberts, Koen \& Shanks, David) 43-92 (Psychology Press, 1997).

7. Rips, L. J., Smith, E. E. \& Medin, D. L. Concepts and categories: Memory, meaning, and metaphysics. in The Oxford Handbook of Thinking and Reasoning (eds. Holyoak, K. J. \& Morrison, R. G.) 177-209 (Oxford University Press, 2012).

8. Rogers, T. T. \& McClelland, J. L. Semantic cognition: A parallel distributed processing approach. (MIT press, 2004).

9. Goldstone, R. L. \& Son, J. Y. Similarity. in The Oxford Handbook of Thinking and Reasoning (eds. Holyoak, K. J. \& Morrison, R. G.) 155-176 (Oxford University Press, 2012).

10. Kriegeskorte, N. \& Kievit, R. A. Representational geometry: integrating cognition, computation, and the brain. Trends Cogn. Sci. 17, 401-412 (2013).

11. Caramazza, A. \& Shelton, J. R. Domain-specific knowledge systems in the brain: The animate-inanimate distinction. J. Cogn. Neurosci. 10, 1-34 (1998).

12. Chao, L. L., Haxby, J. V. \& Martin, A. Attribute-based neural substrates in temporal cortex for perceiving and knowing about objects. Nat. Neurosci. 2, 913-919 (1999).

13. Konkle, T. \& Oliva, A. Canonical visual size for real-world objects. J. Exp. Psychol. Hum. Percept. Perform. 37, 23-37 (2011).

14. Murphy, G. The big book of concepts. (MIT press, 2004).

15. McRae, K., Cree, G. S., Seidenberg, M. S. \& McNorgan, C. Semantic feature production norms for a large set of living and nonliving things. Behav. Res. Methods 37, 547-559 (2005).

16. Devereux, B. J., Tyler, L. K., Geertzen, J. \& Randall, B. The Centre for Speech, Language and the Brain (CSLB) concept property norms. Behav. Res. Methods 46, 1119-1127 (2014). 
17. Hebart, M. N. et al. THINGS: A database of 1,854 object concepts and more than 26,000 naturalistic object images. PLoS ONE 14, e0223792 (2019).

18. Tversky, A. Features of similarity. Psychol. Rev. 84, 327-352 (1977).

19. Barsalou, L. W. Context-independent and context-dependent information in concepts. Mem. Cognit. 10, 82-93 (1982).

20. Maddox, W. T. \& Ashby, F. G. Comparing decision bound and exemplar models of categorization. Percept. Psychophys. 53, 49-70 (1993).

21. Hoyer, P. O. Modeling receptive fields with non-negative sparse coding. Neurocomputing 52, 547-552 (2003).

22. Murphy, B., Talukdar, P. \& Mitchell, T. Learning effective and interpretable semantic models using non-negative sparse embedding. in Proceedings of COLING 2012 1933-1950 (2012).

23. Shepard, R. N. Stimulus and response generalization: A stochastic model relating generalization to distance in psychological space. Psychometrika 22, 325-345 (1957).

24. Kobak, D. \& Berens, P. The art of using t-SNE for single-cell transcriptomics. Nat. Commun. 10, 1-14 (2019).

25. Shelton, J. R., Fouch, E. \& Caramazza, A. The selective sparing of body part knowledge: A case study. Neurocase 4, 339-351 (1998).

26. Pedersen, T., Patwardhan, S. \& Michelizzi, J. WordNet::Similarity - Measuring the relatedness of concepts. in HLT-NAACL 2004: Demonstration papers (eds. Dumais, S., Marcu, D. \& Roukos, S.) 38-41 (ACL Press, 2004).

27. Warrington, E. K. \& Shallice, T. Category specific semantic impairments. Brain 107, 829853 (1984).

28. Rips, L. J. Similarity, typicality, and categorization. in Similarity and analogical reasoning (eds. Vosniadou, Stella \& Ortony, Andrew) 21-59 (Cambridge University Press, 1989).

29. Smith, E. E. \& Sloman, S. A. Similarity- versus rule-based categorization. Mem. Cognit. 22, 377-386 (1994). 
30. Pilehvar, M. T. \& Collier, N. De-conflated semantic representations. in 2016 Conference on Empirical Methods in Natural Language Processing (EMNLP) 1680-1690 (2016).

31. Nosofsky, R. M., Sanders, C. A., Meagher, B. J. \& Douglas, B. J. Toward the development of a feature-space representation for a complex natural category domain. Behav. Res. Methods 50, 530-556 (2018).

32. Nosofsky, R. M., Sanders, C. A., Meagher, B. J. \& Douglas, B. J. Search for the missing dimensions: Building a feature-space representation for a natural-science category domain. Comput. Brain Behav. 3, 13-33 (2020).

33. Zheng, C. Y., Pereira, F., Baker, C. I. \& Hebart, M. N. Revealing interpretable object representations from human behavior. Preprint at arXiv https://arxiv.org/abs/1901.02915 (2019).

34. Keil, F. C. Constraints on knowledge and cognitive development. Psychol. Rev. 88, 187227 (1981).

35. Shepard, R. N. The analysis of proximities: Multidimensional scaling with an unknown distance function. I. Psychometrika 27, 125-140 (1962).

36. Torgerson, W. S. Multidimensional scaling: I. Theory and method. Psychometrika 17, 401419 (1952).

37. Thurstone, L. L. Multiple factor analysis. Psychol. Rev. 38, 406-427 (1931).

38. Tranel, D., Logan, C. G., Frank, R. J. \& Damasio, A. R. Explaining category-related effects in the retrieval of conceptual and lexical knowledge for concrete entities: Operationalization and analysis of factors. Neuropsychologia 35, 1329-1339 (1997).

39. Shepard, R. N. \& Arabie, P. Additive clustering: Representation of similarities as combinations of discrete overlapping properties. Psychol. Rev. 86, 87-123 (1979).

40. Navarro, D. J. \& Lee, M. D. Common and distinctive features in stimulus similarity: A modified version of the contrast model. Psychon. Bull. Rev. 11, 961-974 (2004).

41. Carlson, T. A., Ritchie, J. B., Kriegeskorte, N., Durvasula, S. \& Ma, J. Reaction time for 
object categorization is predicted by representational distance. J. Cogn. Neurosci. 26, 132 142 (2013).

42. Yee, E. \& Thompson-Schill, S. L. Putting concepts into context. Psychon. Bull. Rev. 23, 1015-1027 (2016).

43. Charest, I., Kievit, R. A., Schmitz, T. W., Deca, D. \& Kriegeskorte, N. Unique semantic space in the brain of each beholder predicts perceived similarity. Proc. Natl. Acad. Sci. 111, 14565-14570 (2014).

44. De Haas, B., lakovidis, A. L., Schwarzkopf, D. S. \& Gegenfurtner, K. R. Individual differences in visual salience vary along semantic dimensions. Proc. Natl. Acad. Sci. 116, 11687-11692 (2019).

45. Peterson, J. C., Abbott, J. T. \& Griffiths, T. L. Evaluating (and improving) the correspondence between deep neural networks and human representations. Cogn. Sci. $\mathbf{4 2}$, 2648-2669 (2018).

46. Rajalingham, R. et al. Large-scale, high-resolution comparison of the core visual object recognition behavior of humans, monkeys, and state-of-the-art deep artificial neural networks. J. Neurosci. 38, 7255-7269 (2018).

47. Jozwik, K. M., Kriegeskorte, N., Storrs, K. R. \& Mur, M. Deep convolutional neural networks outperform feature-based but not categorical models in explaining object similarity judgments. Front. Psychol. 8, 1726 (2017).

48. Iordan, M. C., Giallanza, T., Ellis, C. T., Beckage, N. \& Cohen, J. D. Context Matters: Recovering Human Semantic Structure from Machine Learning Analysis of Large-Scale Text Corpora. Preprint at arXiv https://arxiv.org/abs/1910.06954 (2019).

49. Bauer, A. J. \& Just, M. A. Neural representations of concept knowledge. in The Oxford Handbook of Neurolinguistics (eds. de Zubicaray, G. I. \& Schiller, N. O.) 518-547 (Oxford University Press, 2019).

50. Binder, J. R. et al. Toward a brain-based componential semantic representation. Cogn. 
Neuropsychol. 33, 130-174 (2016).

51. Huth, A. G., Nishimoto, S., Vu, A. T. \& Gallant, J. L. A continuous semantic space describes the representation of thousands of object and action categories across the human brain.

Neuron 76, 1210-1224 (2012).

52. Cichy, R. M., Kriegeskorte, N., Jozwik, K. M., van den Bosch, J. J. \& Charest, I. The spatiotemporal neural dynamics underlying perceived similarity for real-world objects. Neurolmage 194, 12-24 (2019).

53. Bankson, B. B., Hebart, M. N., Groen, I. I. A. \& Baker, C. I. The temporal evolution of conceptual object representations revealed through models of behavior, semantics and deep neural networks. Neurolmage 178, 172-182 (2018).

54. Abadi, M. et al. Tensorflow: A system for large-scale machine learning. in 12th Symposium on Operating Systems Design and Implementation 265-283 (2016).

55. Kingma, D. P. \& Ba, J. Adam: A method for stochastic optimization. Preprint at arXiv https://arxiv.org/abs/1412.6980 (2015).

\section{Acknowledgments}

We would like to thank Anna Corriveau for help with data collection in the laboratory experiment and lan Charest, Bradley Love, Alex Martin, and Patrick McClure for helpful discussions and/or comments on the manuscript. This research was supported by the Intramural Research Program of the National Institutes of Health (ZIA-MH-002909), under National Institute of Mental Health Clinical Study Protocol 93-M-1070 (NCT00001360). The funders had no role in study design, data collection and analysis, decision to publish or preparation of the manuscript.

\section{Author contributions}

M.N.H. and C.I.B. conceived and designed the study; M.N.H. collected the data; C.Y.Z., M.N.H. and F.P. designed the computational model; M.N.H., C.Y.Z. and F.P. analyzed the data; M.N.H., C.I.B., F.P., and C.Y.Z. wrote the manuscript and provided critical revisions.

\section{Competing interests}

The authors declare no competing interests. 


\section{Extended Data}

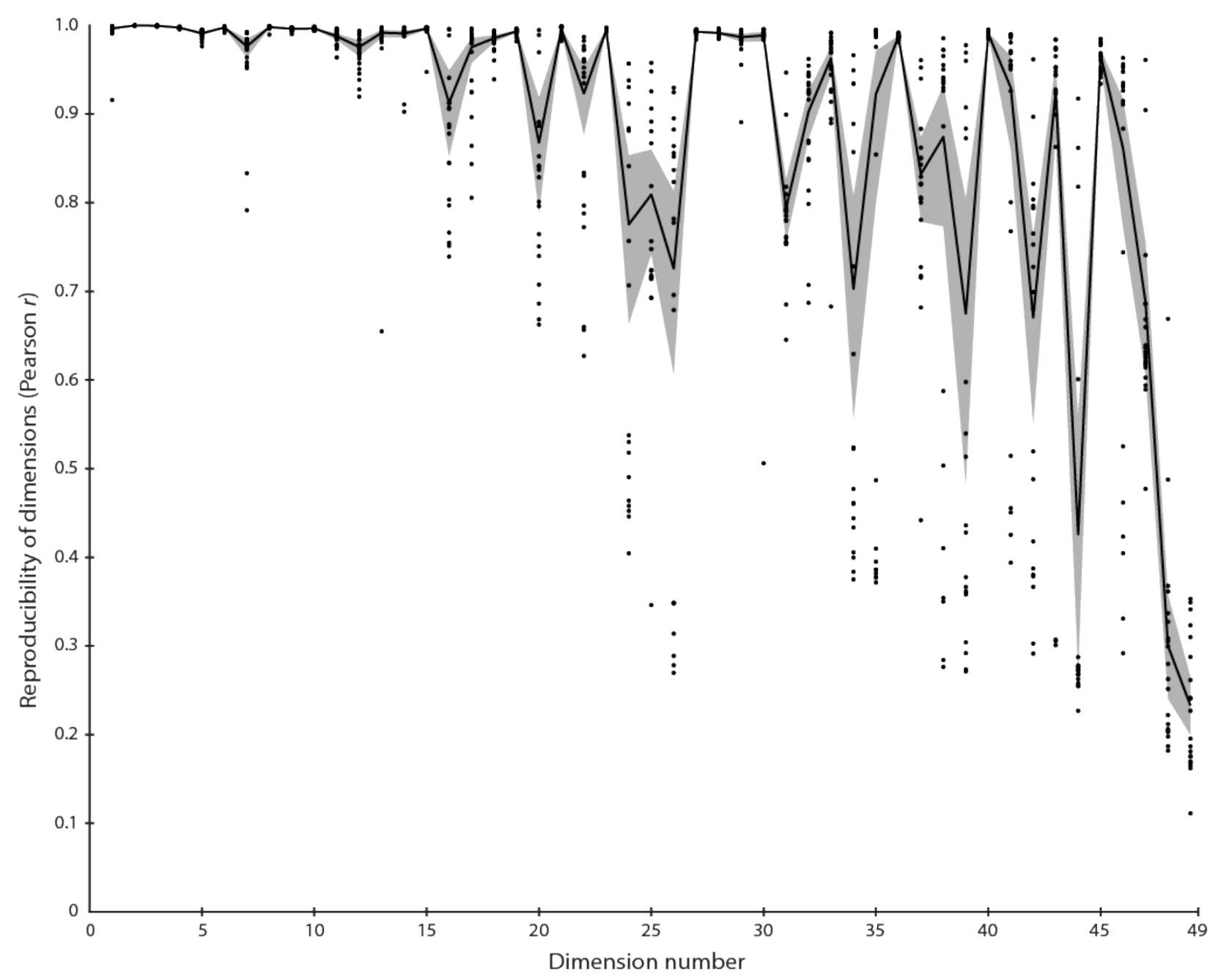

Extended Data Figure 1 | Reproducibility of dimensions in the chosen 49-dimensional embedding across 20 random initializations (see Extended Data Figure 2 for a list of all dimension labels). Shaded areas reflect $95 \%$ confidence intervals. 


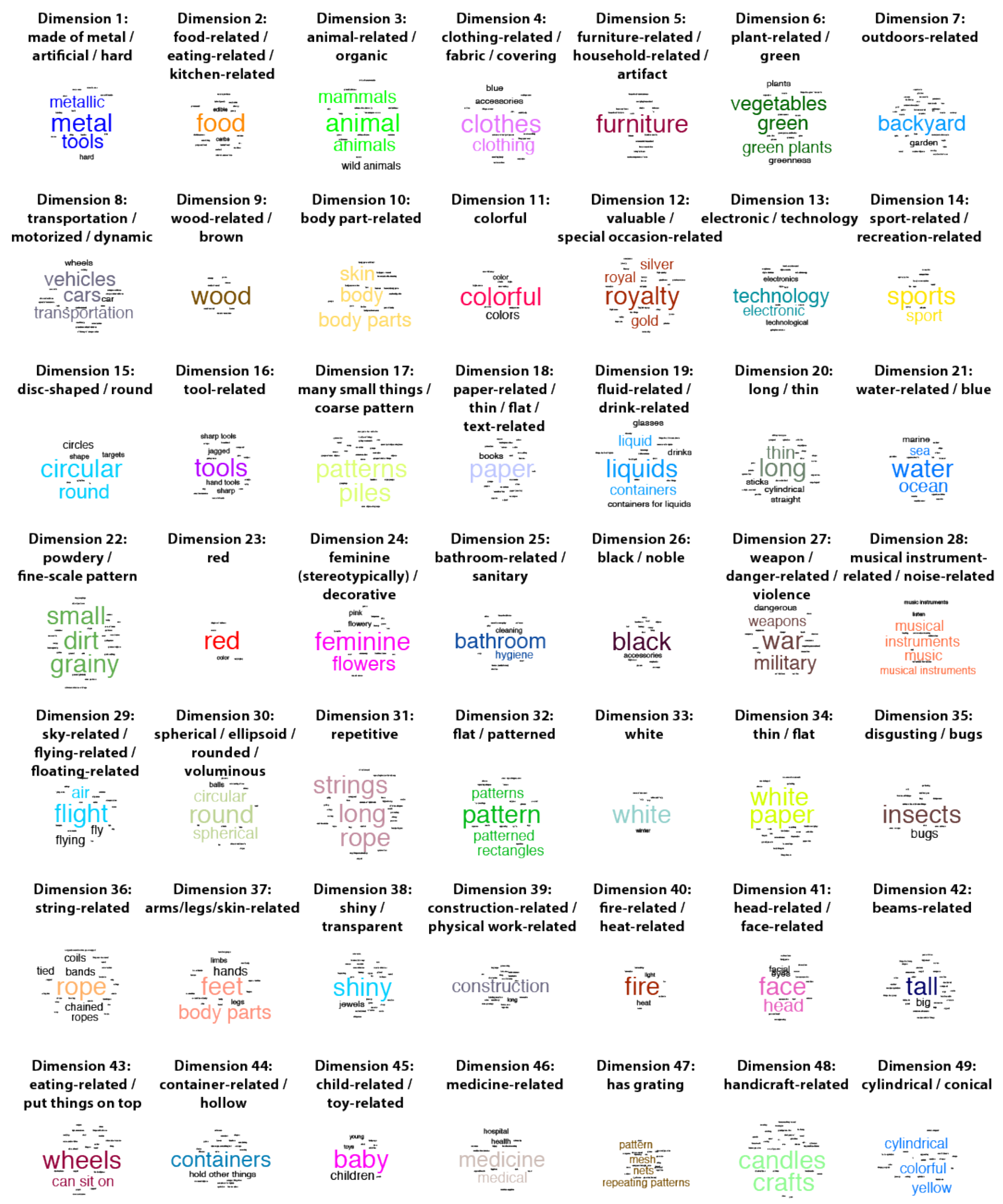

Extended Data Figure 2 | Labels for all 49 dimensions, with respective word clouds reflecting the naming frequency across 20 participants. The dimensions appear to reflect both perceptual and conceptual properties of objects. A visual comparison between labels and word clouds indicates a generally good agreement between participant naming and the labels we provided for the dimensions. 


\begin{tabular}{|c|c|c|c|c|c|}
\hline Dimension name & $\begin{array}{l}\text { Category } \\
\text { name }\end{array}$ & $\begin{array}{l}\text { Number of } \\
\text { objects in } \\
\text { category }\end{array}$ & $\begin{array}{l}\text { Spearman's } r \\
\quad \pm 95 \% \mathrm{Cl}\end{array}$ & $\begin{array}{c}p \text {-value } \\
\text { (uncorrected) }\end{array}$ & $\begin{array}{c}p \text {-value } \\
\text { (FDR-corrected) }\end{array}$ \\
\hline $\begin{array}{l}\text { weapon / danger- } \\
\text { related }\end{array}$ & weapon & 48 & $\begin{array}{l}\text { Spearman's } r \\
(90 \% \mathrm{Cl})\end{array}$ & $<0.001$ & $<0.001$ \\
\hline $\begin{array}{c}\text { transportation / } \\
\text { dynamic }\end{array}$ & vehicle & 70 & $\begin{array}{r}0.62 \\
(0.43-0.76)\end{array}$ & $<0.001$ & $<0.001$ \\
\hline furniture-related & furniture & 38 & $\begin{array}{r}0.62 \\
(0.45-0.75)\end{array}$ & $<0.001$ & $<0.001$ \\
\hline $\begin{array}{l}\text { electronic / } \\
\text { technology }\end{array}$ & $\begin{array}{l}\text { electronic } \\
\text { device }\end{array}$ & 74 & $\begin{array}{r}0.61 \\
(0.45-0.74)\end{array}$ & $<0.001$ & $<0.001$ \\
\hline animal-related & animal & 177 & $\begin{array}{r}0.60 \\
(0.45-0.71)\end{array}$ & $<0.001$ & $<0.001$ \\
\hline sport-related & $\begin{array}{c}\text { sports } \\
\text { equipment }\end{array}$ & 63 & $\begin{array}{r}0.58 \\
(0.48-0.67)\end{array}$ & $<0.001$ & $<0.001$ \\
\hline clothing-related & clothing & 108 & $\begin{array}{r}0.53 \\
(0.34-0.68)\end{array}$ & $<0.001$ & $<0.001$ \\
\hline $\begin{array}{l}\text { fluid-related / } \\
\text { drink-related }\end{array}$ & drink & 19 & $\begin{array}{r}0.52 \\
(0.39-0.62)\end{array}$ & 0.026 & 0.034 \\
\hline food-related & food & 294 & $\begin{array}{r}0.46 \\
(0.04-0.74)\end{array}$ & $<0.001$ & $<0.001$ \\
\hline child/toy-related & toy & 34 & $\begin{array}{r}0.42 \\
(0.33-0.50)\end{array}$ & 0.016 & 0.024 \\
\hline instrument-related & $\begin{array}{c}\text { musical } \\
\text { instrument }\end{array}$ & 33 & $\begin{array}{r}0.37 \\
(0.04-0.63)\end{array}$ & 0.023 & 0.033 \\
\hline body part-related & body part & 34 & $\begin{array}{r}0.35 \\
(0.08-0.58)\end{array}$ & 0.030 & 0.036 \\
\hline
\end{tabular}




\begin{tabular}{|c|c|r|r|r|r|} 
medicine-related & $\begin{array}{c}\text { medical } \\
\text { equipment }\end{array}$ & 27 & $\begin{array}{r}0.33 \\
(0.04-0.56)\end{array}$ & 0.052 & 0.059 \\
\hline tool-related & tool & 107 & $\begin{array}{r}0.32 \\
(-0.09-0.64)\end{array}$ & 0.002 & 0.004 \\
\hline $\begin{array}{c}\text { container-related / } \\
\text { hollow }\end{array}$ & container & 105 & $\begin{array}{r}0.28 \\
(0.14-0.41)\end{array}$ & 0.004 & 0.007 \\
\hline $\begin{array}{c}\text { insects / } \\
\text { disgusting }\end{array}$ & insect & 17 & $(0.10-0.40)$ & 0.245 & 0.261 \\
\hline $\begin{array}{c}\text { plant-related / } \\
\text { green }\end{array}$ & plant & 47 & $(-0.25-0.55)$ & 0.18 & 0.688 \\
\hline
\end{tabular}

Extended Data Table 1 | Detailed results of inferential statistical analyses correlating categoryrelated dimensions with typicality of their category. One-sided $p$-values were generated using randomization tests and were controlled for false discovery rate (FDR) across multiple tests. $90 \%$ confidence intervals were used to complement one-sided tests. 